\title{
Regulatory roles for mitochondria in the peri-implantation mouse blastocyst: possible origins and developmental significance of differential $\Delta \Psi \mathrm{m}$
}

\author{
Jonathan Van Blerkom, Heather Cox and Patrick Davis \\ Department of Molecular, Cellular and Developmental Biology, University of Colorado, Boulder, \\ Colorado 80309, USA
}

Correspondence should be addressed to J Van Blerkom; Email: vanblerk@buffmail.colorado.edu

\begin{abstract}
Studies of mitochondria in mouse and human oocytes and preimplantation stage embryos have focused primarily on their metabolic capacity to generate ATP. However, it is becoming increasingly apparent that mitochondria are also regulatory agents in other processes involved in the establishment of developmental competence, including calcium homeostasis and apoptosis. The magnitude of the inner mitochondrial membrane potential, or its polarity $(\Delta \Psi \mathrm{m})$, is a physiochemical property of mitochondria related to levels of organelle activity, and differences in the magnitude and spatial distribution of high- and low-polarized mitochondria have been suggested to influence oocyte and early embryo competence. Here, we investigated mitochondrial polarity in normal and diapausing peri-implantation-stage mouse blastocysts, and their corresponding outgrowths, for indications of cell-type-specific regulatory functions or activities in which these organelles may be engaged. The results demonstrate that cell-type- and location-specific domains of differential $\Delta \Psi \mathrm{m}$ exist in the peri-implantation blastocyst and remain unchanged during blastocyst outgrowth and during delayed implantation, which for the latter, is accompanied by the suppression of mitochondrial oxidative phosphorylation. Our findings demonstrate that cell-type-specific $\Delta \Psi \mathrm{m}$ in the peri-implantation blastocyst is not an intrinsic property of the corresponding mitochondria but one that can be mediated by the dynamics of intercellular contact. Cells with high- or low-polarized mitochondria are differentially affected by photosensitization, with developmental consequences related to embryo behavior and outgrowth performance. Differences in polarity are discussed with respect to the participation of mitochondria in regulatory and morphogenetic processes in the normal periimplantation embryo. The persistence of high $\Delta \Psi \mathrm{m}$ in the diapausing embryo is suggested to be associated with the regulation of levels of cytoplasmic free calcium and the ability of the embryo to reactivate development when delayed implantation terminates.

Reproduction (2006) 131 961-976
\end{abstract}

\section{Introduction}

It has long been known that mitochondria, by virtue of their ability to generate ATP, have a central role in the normality of early mammalian development (reviewed by Biggers \& Borland 1976, Van Blerkom \& Motta 1979). This conclusion is supported by recent studies (reviewed by Cummins 2002, Brenner 2004) in which metabolic, structural and numerical (organelle and mitochondria DNA copy number) defects have been be associated with maturational failure for the oocyte and premature arrest or abnormal development for the embryo (Van Blerkom et al. 1995, 2000, Müller-Höcker et al. 1996, Steuerwald et al. 2000, Reynier et al. 2001, Brenner 2004). However, whether mitochondria have a regulatory role in early development that is distinct from their metabolic contribution has only recently been considered with respect to other functions, such as their ability to sequester and release calcium, modify proteins or initiate apoptosis (reviewed by Van Blerkom 2004).

The magnitude of the potential difference (mitochondrial polarity) across the inner mitochondrial membrane $(\Delta \Psi \mathrm{m})$ is a electro-chemical property of these organelles that can be estimated in living cells with reporter stains whose fluorescent emission characteristics are potential (potentiometric) sensitive, such as JC-1 (Reers et al. 1995). Differences in $\Delta \Psi \mathrm{m}$ detected within and between cultured cells have been largely thought to reflect corresponding differences in mitochondrial function or levels of activity (Cossarizza et al. 1996, Salvioli et al. 1977, Dedov \& Roufogalis 1999, Dias et al. 1999). In the mouse 
and human oocyte and early embryo, high- and lowpolarized mitochondria have a distinct spatial distribution in the pericortical/subplasmalemmal and perinuclear domains respectively (Van Blerkom et al. 2002). Changes in spatial distribution or polarity (high to low, or vice versa) detected in fresh (Wilding et al. 2001, 2003, Van Blerkom et al. 2002, 2003, Acton et al. 2004) and thawed (Ahn et al. 2002, Jones et al. 2004) metaphase II oocytes and embryos have been associated with developmental incompetence or abnormality. These findings suggest that the analysis of $\Delta \Psi \mathrm{m}$ may be a means by which differential mitochondrial activity or regulatory function(s) can be investigated in living preimplantation-stage embryos, especially with respect to domains of high- and low-polarized organelles (Van Blerkom 2004).

Van Blerkom et al. (2002) reported that high-polarized mitochondria occur in the trophectoderm, but not the inner cell mass (ICM), of expanded mouse and human blastocysts. Here, mitochondrial polarity was examined in normal, diapausing and JC-1-photosensitized, hatched (peri-implantation) mouse blastocysts, and their respective in vitro outgrowths. We intended to investigate how location- and cell-type-specific $\Delta \Psi \mathrm{m}$ occurs and whether it indicates different regulatory functions for mitochondria. The results extend our earlier findings and show that the magnitude of $\Delta \Psi \mathrm{m}$ is determined by extrinsic forces and may reflect different regulatory roles for mitochondria during the peri-implantation stage.

\section{Materials and Methods}

\section{Recovery of normal and delayed implanted mouse blastocysts}

Superovulated mice were ovariectomized on day 2.5 of pregnancy (detection of vaginal plug $=$ day 1 ) and given a single subcutaneous injection of Depo-Provera $(1.0 \mathrm{mg})$. Chemical ovariectomy followed the protocol of MacLeanHunter and Evans (1999), in which the estrogen antagonist/ agonist tamoxifen (10 $\mu \mathrm{g}$; Sigma) and Depo-Provera (1.0 mg; Pharmacia and Upjohn, Kalamazoo, MI, USA) were coadministered on day 2.5. Hatched blastocysts were recovered from untreated animals on day 4.5 and from treated animals on days $1-7$ of delay by gently flushing the uterus with HEPES-buffered medium (M2 or HTF) supplemented with $1 \%$ BSA.

\section{Blastocyst outgrowth}

Outgrowths of normal and delayed implanted embryos occurred on glass cover slips in high-glucose-containing DMEM supplemented with essential amino acids, glutamine (GlutaMax) and 20\% fetal bovine serum (Invitrogen), conditions previously shown to support activation from delay and robust trophectoderm outgrowth and expansion in vitro (Van Blerkom et al. 1979, Van Blerkom \& Chavez 1981). After 1.5 days of culture, the colonies resulting from normal and delayed implanted embryos were characterized by a highly flattened circumferential monolayer of outgrowing mural trophectoderm that surrounded a central mass of ICM cells. The ICM-containing central cores of the outgrowths were dissected from the mural trophoblast with glass needles, exposed to trypsin $(30 \mathrm{~min}$, $0.25 \%$ ) in calcium- and magnesium-free PBS with continual agitation and pipetting, and replated. A similar protocol was followed for JC-1-stained blastocysts illuminated in the FITC or RITC channels (see below). From three to five ICM masses were treated with trypsin, as described above, and replated. Proliferating ICM cells were restained with JC- 1 at $6 \mathrm{~h}$ and on days $1-7$ of culture, and examined by fluorescence microscopy. Undisturbed outgrowths were stained with JC-1 (see below) on days 1-5 of culture.

\section{DNA and mitochondrial staining}

Immediately after recovery, representative normal and delayed implanted blastocysts were fixed in PBS containing $3.7 \%$ formaldehyde for $15-20 \mathrm{~min}$, and after washing in HTF supplemented with $1 \%$ BSA, they were exposed to the DNA-specific fluorescent stain DAPI (4',6-diamidino-2phenylindole) $10 \mu \mathrm{g} / \mathrm{ml}$ for $15 \mathrm{~min}$ ) (Sigma). Living embryos were exposed to the following mitochondriaspecific fluorescent probes (Molecular Probes, Eugene, OR, USA) (Van Blerkom et al. 2002): rhodamine 123 (r123, $10 \mu \mathrm{g} / \mathrm{ml}, 30 \mathrm{~min})$, MitoTracker Orange (MO, $200 \mathrm{nmol} / \mathrm{l}$, $30 \mathrm{~min})$ or JC-1 (5,5'6,6'-tetrachloro-1,1,3,3'-tetraethylbenzimidazolycarbocyanine iodide $(1 \mu \mathrm{g} / \mathrm{ml}, 30 \mathrm{~min})$. In other studies, normal and diapausing blastocysts were either mechanically collapsed by passage through a narrow-bore micropipette and stained with mitochondria-specific probes during re-expansion, or manipulated with glass needles such that a significant rent was made in the mural trophectoderm, directly exposing the ICM to the stains. Representative embryos and outgrowths were exposed to the $\Delta \Psi \mathrm{m}$-collapsing proton ionophore FCCP (carbonyl cyanide $p$-(trifluoromethoxy)phenyl-hydrazone (Sigma), $10 \mu \mathrm{g} / \mathrm{ml}$, for $10 \mathrm{~min}$ before JC-1 staining. DNA analysis and mitochondrial fluorescence used conventional epifluorescence (EF) and scanning laser confocal microscopy (SLCM), as previously described (Van Blerkom et al. 2002, 2003, Jones et al. 2004).

\section{BrdU staining}

Normal and delayed implanted blastocysts and outgrowths were incubated in modified MEM containing $10 \mu \mathrm{M}$ BrdU (5-bromo-2'-deoxyuridine) for a minimum of $4 \mathrm{~h}$ and a maximum of $24 \mathrm{~h}$, followed by fixation and antibody staining, according to the manufacturer's recommendations (In Situ Cell Proliferation Kit, FLUOS Assay; Roche). Briefly, intact embryos and outgrowths were washed in PBS, fixed at $4^{\circ} \mathrm{C}$ in a solution of $70 \% \mathrm{EtOH}$ and $30 \% 50 \mathrm{mM}$ glycine $(\mathrm{pH} \mathrm{7)}$ for $30 \mathrm{~min}$ at room 
temperature in PBS containing 0.1\% Tween-20 (permeabilization buffer), and denatured in $4 \mathrm{M} \mathrm{HCl}$ for $10 \mathrm{~min}$. Samples were washed several times in the incubation buffer (also provided with the kit) followed by 30-min incubation in the blocking solution provided with the kit. Washed samples were incubated in the presence of a fluorescein-conjugated, anti-BrdU monoclonal antibody for $45 \mathrm{~min}$ in a humidified chamber maintained at $37^{\circ} \mathrm{C}$. Samples were washed in permeabilization buffer for $20 \mathrm{~min}$ and counterstained for $15 \mathrm{~min}$ in DAPI $(10 \mu \mathrm{g} / \mathrm{ml})$.

\section{Photosensitization}

Normal day-5 blastocysts were stained with JC-1 and exposed to ultraviolet illumination $(100 \mathrm{~W})$ in either the FITC or the RITC channel for up to $60 \mathrm{~s}$ with a $\times 40$ fluorescence lens. After exposure, blastocysts were returned to culture and assessed for ATP content, BrdU incorporation, DNA fragmentation and oxidation, and outgrowth behavior and performance in vitro at timed intervals (see below). From preliminary studies, a standard exposure of $8 \mathrm{~s}$ was found to have differential effects on blastocyst activities that may be related to mitochondrial function.

\section{DNA oxidation and fragmentation analysis}

The detection of 8-deoxyoxoguanine has been used to assess quantitatively levels of oxidative damage to DNA, such as may result from the production of reactive oxygen species (e.g. superoxide) by structurally damaged or functionally compromised mitochondria (Struthers et al. 1998), that may occur after photosensitization. After illumination in the FITC or RITC channels, blastocysts were cultured for $24 \mathrm{~h}$, washed in modified MEM, fixed in a PBS containing $3.7 \%$ formaldehyde $(\mathrm{pH} \mathrm{7.4)}$ for $20 \mathrm{~min}$, and permeabilized and stained with Texas Red Avidin (1:200 dilution; Molecular Probes), as described by Radisky et al. (2005). Positive controls were generated by exposure to peroxide $(100 \mu \mathrm{M} / \mathrm{I}$ in PBS) for $30 \mathrm{~min}$. The level of DNA fragmentation in normal, delayed implanted and photosensitized, JC-1-stained blastocysts was examined by whole-mount fluorescence microscopy after terminal deoxynucleotidyl transferase-mediated dUTP nick end-labeling (TUNEL), as previously described (Van Blerkom et al. 2001).

\section{ATP content analysis}

Measurements of net average ATP content followed a previously described protocol (Van Blerkom et al. 1995). Briefly, single blastocysts were rapidly frozen to $-80^{\circ} \mathrm{C}$ in $200 \mu \mathrm{l}$ ultrapure water. ATP levels were quantified by measuring the luminescence (Berthold LB 9501 luminometer, Berthold Technologies, Oak Ridge, TN, USA) generated in an ATP-dependent, luciferin-luciferase bioluminescence assay (Bioluminescence Somatic Cell Assay System; Sigma). A standard curve containing 14 ATP concentrations from $1 \mathrm{fmol}$ to 5 pmol was generated for each series of analyses. Differences in cell number and ATP content were analyzed statistically by the unpaired $t$ test (two-sample) with values considered significant at $P<0.01$.

\section{Time-lapse microscopy}

Hatched blastocysts that were untreated after recovery, recovered from the uterus on day 5 of diapause, and stained with JC-1 and illuminated in the FITC or RITC channel were cultured in outgrowth medium and imaged by time-lapse videomicroscopy at 5 -min intervals. Cultures were maintained at $37^{\circ} \mathrm{C}$, and the timed behavior of embryos was recorded on a laser disk, as previously described (Van Blerkom et al. 2001).

\section{Results \\ Nuclear and mitochondrial staining of normal and delayed implanted mouse blastocysts}

In preliminary studies, comparable patterns and intensities of fluorescent staining with DNA- and mitochondriaspecific probes were observed in blastocysts on days 1,3 and 5 of diapause with delayed implantation induced by surgical or chemical ovariectomy. Consequently, approximately $75 \%$ of the delayed implanted embryos examined in this study were obtained after chemical ovariectomy. Seventy day-4.5 blastocysts and a minimum of 30 embryos on days 1, 2, 3 and 5 of delayed implantation were stained with DNA- or mitochondria-specific fluorescent probes. Twenty-three and 12 embryos respectively were stained with JC- 1 on days 6 and 7 of diapause.

Cell numbers in normal (Fig. 1A) and delayed implanted blastocysts (e.g. day 3; Fig. 1G) were derived from nuclear counts in representative embryos stained with DAPI, and were analyzed with pseudocolor imaging by scanning laser confocal microscopy (SLCM). Differences in average cell numbers were evident when normal day- 4.5 blastocysts $(119 \pm 11 ; n=29)$ were compared with embryos on days $1(127 \pm 9 ; n=27)$ and $3(131 \pm 7 ; n=31)$ of delay, and these differences were significant. There were no further increases in cell numbers beyond day 3 . These findings agree with previous cell number determinations (Chavez \& Van Blerkom 1979).

\section{Mitochondrial staining of normal and delayed implanted blastocysts}

Representative hatched blastocysts from cohorts recovered on day 4.5 of pregnancy and on days 1, 2, 3, 5 and 6 of delay were examined by conventional epifluorescence (EF) or SLCM after staining with $\mathrm{MO}(n=16$; e.g. Fig. 1B, normal day 4.5) or $\mathrm{r} 123$ ( $n=28$; e.g. Fig. $1 \mathrm{C}$, day-5 delay, shown in the FITC channel). Mitochondrial staining was clearly detectable in the ICM and trophectoderm of all normal and diapausing embryos. Figure $1 \mathrm{E}$ and $\mathrm{F}$ show the typical pattern of JC- 1 fluorescence in a day- 4.5 blastocyst 

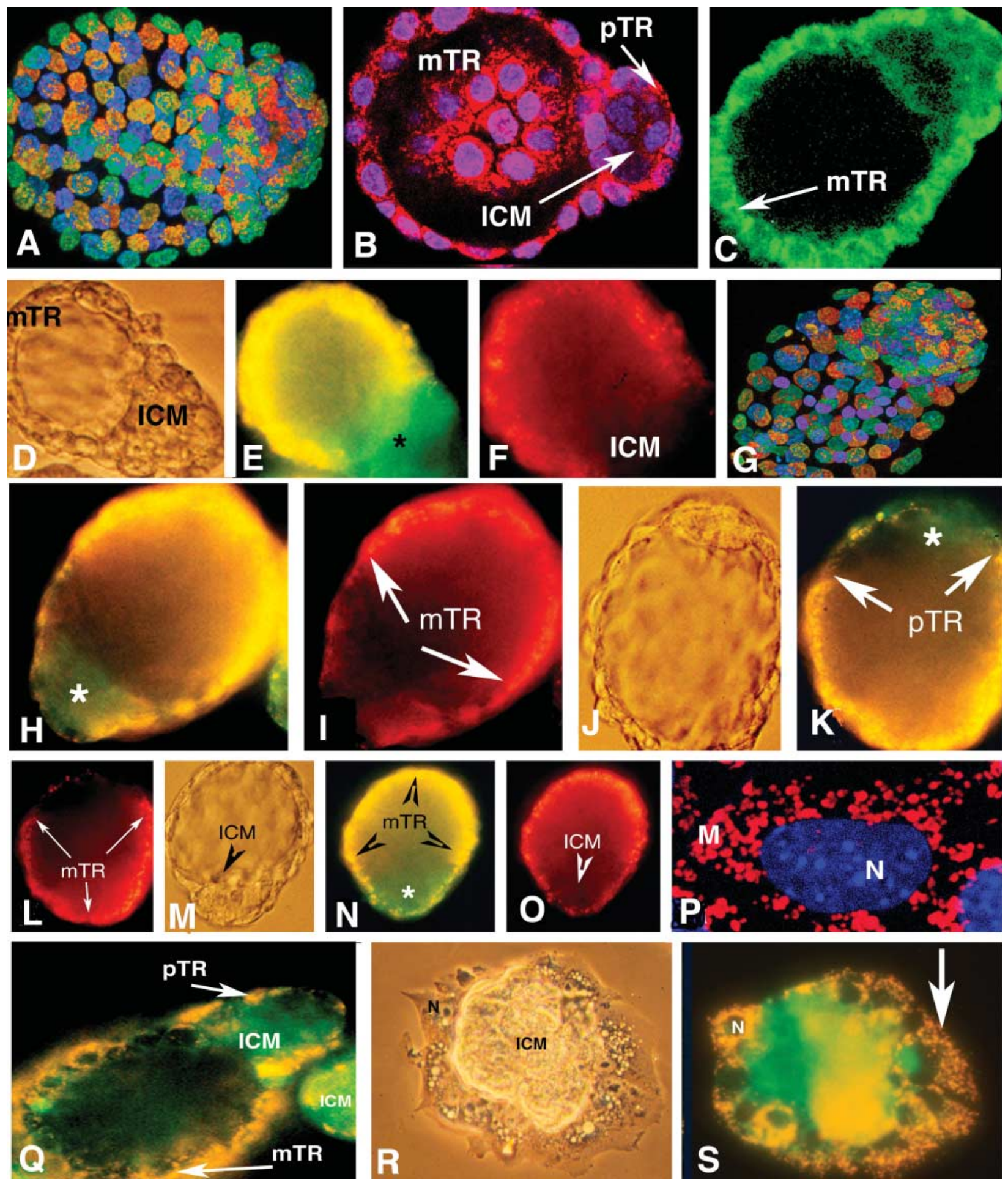

Figure 1 (Panels A and I) Fully compiled, pseudocolor, scanning laser confocal images (SLCM) of DAPI fluorescent nuclei in a normal day-4.5 mouse embryo and a diapausing blastocyst on day 3 of delayed implantation respectively. The following panels show mitochondria staining with MitoTracker Orange (panel B), rhodamine 123 (panel C) and JC-1 fluorescence in the FITC (panels E, H, K, N and Q) and RITC channels (panels F, $\mathrm{I}, \mathrm{L}$ and $\mathrm{O}$ ) in normal (panel D) and delayed implanted, diapausing blastocysts (panels J and M). Perinuclear (N) mitochondrial (M) JC-1, J-aggregate fluorescence in a single mural trophectodermal cell is shown by SLCM in panel P. (Panels D, J and M) Light microscopic images of the blastocysts from which the corresponding patterns of JC- 1 fluorescence noted above were obtained. Differences in the distribution of mitochondrial JC-1 and J-aggregate fluorescence (panel S) in a mouse blastocyst outgrowth on day 1.5 (panel R) indicate cells containing high-polarized red-fluorescent and low-polarized green-fluorescent mitochondria. ICM: inner cell mass; pTR: polar trophectoderm; mTR: mural trophectoderm. 
(Fig. 1D) illuminated in the FITC (Fig. 1E) and RITC channels (Fig. 1F) respectively. As described previously (Van Blerkom et al. 2002), the EF filter system used allows fluorescence from the RITC channel to extend into the FITC channel such that in the FITC channel, JC-1-J-aggregatepositive cells fluoresce bright yellow/orange, and J-aggregate negative cells, such as those in the ICM, fluoresce green (asterisk, Fig. 1E). All day-4.5 embryos $(n=82)$ showed the same pattern of JC-1, J-aggregate fluorescence; the most intense J-aggregate signal occurred in the mural trophectoderm and diminished toward the polar trophectoderm, which exhibited scant punctate J-aggregate fluorescence, when detectable. The absence of detectable J-aggregate fluorescence in the ICM and polar trophectoderm was confirmed in the RITC channel (Fig. 1F). Timed incubations in the presence of JC-1 showed the first evidence of J-aggregate fluorescence in the mural trophoblast after $5 \mathrm{~min}$. With continued staining for up to $3 \mathrm{~h}$, the relative intensity of the J-aggregate signal increased, but its distribution within the embryo remained unchanged. For example, the same pattern of J-aggregatepositive and -negative fluorescence occurred in collapsed blastocysts stained with JC-1 during re-expansion (3-h exposure, Fig. 1Q) and in embryos manipulated to create a rent that continuously exposed the ICM to this probe (image not shown). The ICM portion of a second embryo stained with JC- 1 for $3 \mathrm{~h}$ is located on the far right in Fig. 1Q.

Diapausing embryos stained immediately after recovery showed the same patterns and relative intensities of JC- 1 and J-aggregate fluorescence observed in their day-4.5 counterparts. For example, representative images of JC-1 fluorescence on days $3(n=45), 5(n=22 ;$ Fig. $1 \mathrm{~J})$ and 7 $(n=9$; Fig. $1 \mathrm{M})$ are shown in the FITC channel in Fig. $1 \mathrm{H}$, $\mathrm{K}$ and $\mathrm{N}$, and in the RITC channel in Fig. $1 \mathrm{I}, \mathrm{L}$ and $\mathrm{O}$ respectively. J-aggregate fluorescence was intense in the mural trophectoderm (mTR), scant in the polar trophectoderm (pTR) and undetectable in the ICM (e.g. asterisk; Fig. $1 \mathrm{H}, \mathrm{K}$ and $\mathrm{N}$ ). Figure $1 \mathrm{P}$ is a high-magnification SLCM image of a single mural trophectodermal cell in which compression of the day-3 diapausing embryo between glass cover slips enabled imaging of individual J-aggregate-positive mitochondria (M). As in our previous findings with normal mouse blastocysts (Van Blerkom et al. 2002 ), collapsing $\Delta \Psi \mathrm{m}$ by preincubation in the presence of the proton ionophore FCCP completely abolished detectable trophectodermal J-aggregate fluorescence (image not shown).

\section{JC-1 staining of blastocyst outgrowths}

Comparable patterns and extent of trophectoderm outgrowth (OG) were observed for day-4.5 blastocysts $(n=57)$ and diapausing blastocysts reactivated in vitro on days $3(n=26), 5(n=16)$ and $6(n=19)$ of delayed implantation. As in previous findings (Van Blerkom et al. 1979), a circumferential layer of highly flattened, mural trophectodermal cells surrounded a central core composed primarily of ICM cells (e.g. OG day 1 , Fig. 1R; OG day 1.5 , Fig. 2A). A characteristic spatial and cell-typespecific pattern of mitochondrial polarity was evident during the early stages of outgrowth (Figs $1 \mathrm{~S}$ and 2B); lowpolarized mitochondria (e.g. LP, Fig. 2B) occurred in the centrally located, multilayered mass of ICM cells, while high-polarized mitochondria occurred throughout the cytoplasm of trophectodermal cells (arrow, Fig. 1S; HP, Fig. 2B).

Cell-type-specific differences in mitochondrial polarity persisted between the ICM and trophectodermal compartments of undisturbed blastocyst outgrowths after cytokinesis and expansion of the trophectoderm ceased, around OG days 3-4 (OG day 5, Fig. 2C). However, with continued culture, the spatial distribution that characterized high- and low-polarized mitochondria in trophectodermal cells became less distinct. While high-polarized mitochondria were confined to the margins of these cells (red arrows, Fig. 2D), perinuclear mitochondria were largely low polarized (white arrows, Fig. 2D), unlike the situation observed during earlier phases of active outgrowth and expansion, where perinuclear mitochondria were primarily high polarized (Fig. 2B). The behavior, outgrowth characteristics and differential pattern of JC-1 and J-aggregate fluorescence of the ICM and trophectoderm were identical in day-4.5 blastocysts (Fig. $1 \mathrm{R}$ and S) and diapausing embryos activated in vitro (day 5 of diapause, Fig. 2A and B).

To determine whether the low mitochondrial polarity detected in the ICM of intact blastocysts and undisturbed outgrowths was an intrinsic feature of these cells, ICM cores from day-1.5 outgrowths (asterisk, Fig. 3A; $n=21$ ) were isolated, disaggregated and replated. During the early stages of disaggregation, the ICM cores appeared as clusters of round cells that were loosely connected at the periphery and more densely associated in the interior (Fig. 3B). When stained with JC-1 and imaged by fluorescence microscopy $1.5 \mathrm{~h}$ after disaggregation, some of the loosely associated peripheral cells showed apical J-aggregate fluorescence (red arrows, Fig. 3C), while those in the interior fluoresced green. From three to five disaggregated ICM cores were combined, replated and cultured. The resulting colonies of cells were uniformly small, mononucleated and phenotypically distinct ( $n=12$; e.g. Fig. 3D) from the highly flattened and occasionally multinucleated trophectodermal cells typical of undisturbed blastocyst outgrowths (e.g. Figs $2 \mathrm{~A}$ and $4 \mathrm{G}$ ).

ICM-derived cells showed robust proliferation during 7 days of monitored culture (e.g. day 4 of culture; Fig. 3D). After staining with JC-1, J-aggregate-positive cells were observed throughout the cultures (Fig. 3E), with the exception of high-density portions of the monolayer (asterisk, Fig. 3D), whose fluorescence characteristics indicated the presence of low-polarized mitochondria (asterisk, Fig. 3E). At higher magnifications, cells loosely associated in small clusters (such as cells indicated by a red arrow in Fig. 3E) 

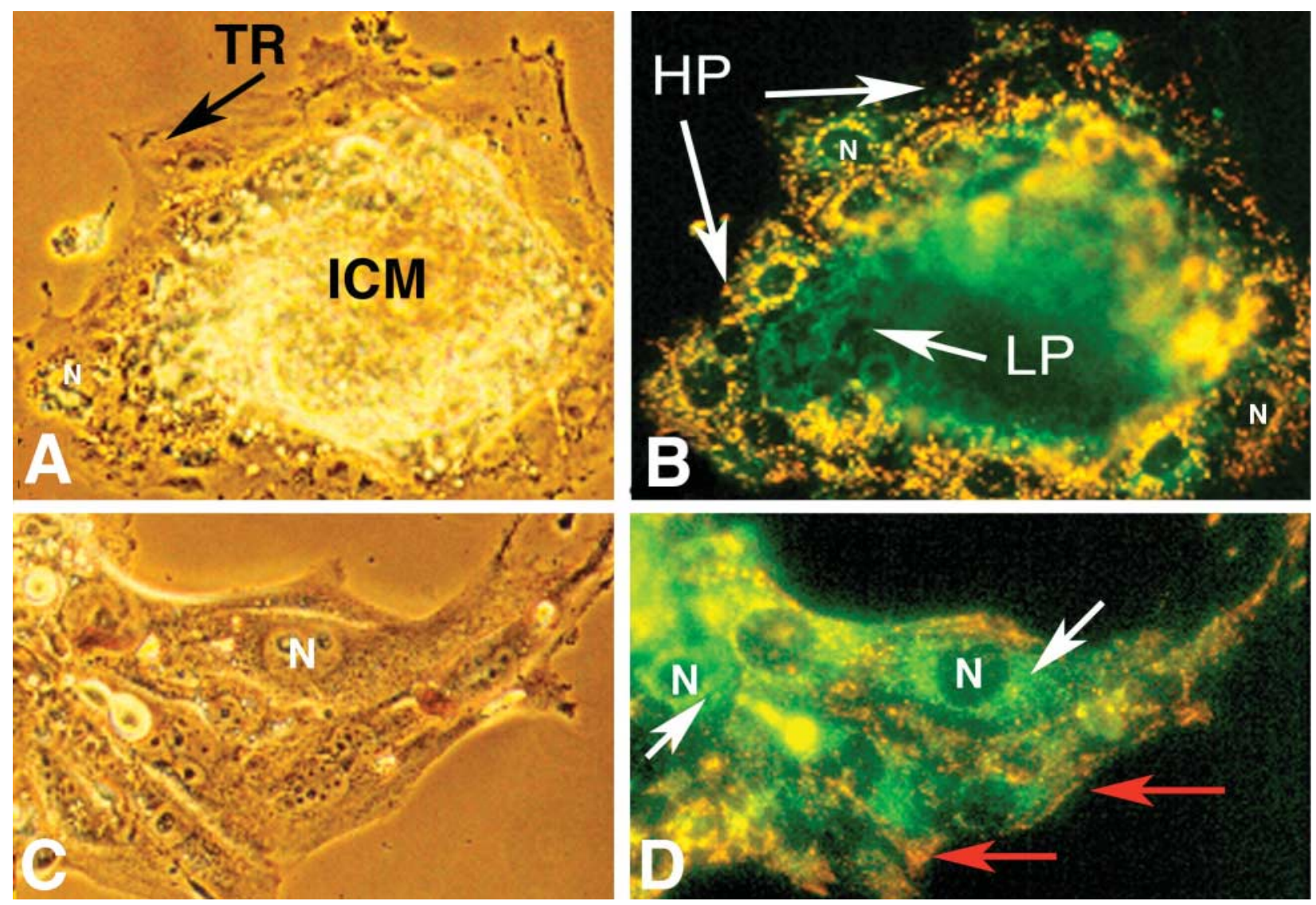

Figure 2 The unique spatial distribution of cells containing high- and low-polarized mitochondria in a blastocyst outgrowth that was derived from an embryo activated from diapause on day 5 of delayed implantation and stained with JC- 1 on day 1.5 of culture (panel A) is shown by fluorescence microscopy in panel B. Typically, orange-red fluorescent, high-polarized mitochondria (HP) are present throughout the cytoplasm of early outgrowing monolayer of trophectodermal cells (TR), while the centrally located, multilayered inner cell mass (ICM) contains low-polarized (LP), green fluorescent mitochondria. However, as these cultures age (day 5, panel C), high-polarized mitochondria in the trophectodermal cells are largely confined to the apical borders whereas low-polarized mitochondria are largely perinuclear $(N$, arrows, panel $D)$.

contained both high- (red arrow, Fig. 3F) and low-polarized mitochondria (green arrow, Fig. 3F). By contrast, isolated cells and cells in low-density regions of the monolayer whose margins were largely free (e.g. blue arrow, Fig. 3E) were uniformly J-aggregate positive (Fig. $3 \mathrm{G})$, indicating that the corresponding population of mitochondria was high polarized.

\section{Photosensitization of JC-1-stained embryos}

The extent to which mitochondrial polarity may be related to cell function and behavior was investigated by JC-1 photosensitization of day-4.5 blastocysts, which were illuminated in the FITC or RITC channels and then returned to culture. Preliminary experiments demonstrated that 8-s exposure had differential and cell-type-specific sublethal effects of developmental significance, and this duration became standard. Normal patterns of BrdU incorporation (which reflects the ability of the cells to replicate DNA) detected by fluorescence microscopy in day- 4.5 blastocysts (Fig. 4A; $n=20$ ) are shown in Fig. 4B, with nuclear staining observed in all embryonic compartments (ICM, mural (MT) and polar trophectoderm (PT)). Blastocyst outgrowths from these embryos (e.g. Fig. 4C, OG day 2) composed of a characteristic ICM core (asterisk) and a highly flattened peripheral trophectodermal monolayer (arrow), routinely showed nuclear BrdU staining throughout the culture (Fig. 4D; $n=26$ ). Exposure of JC-1-stained embryos (Fig. 4E) in the FITC channel $(n=40)$ had no discernible effect on BrdU incorporation (Fig. 4F), the timing or extent of outgrowth (OG day 2, Fig. 4G), or BrdU incorporation (Fig. $4 \mathrm{H}$ ). In contrast, exposure in the RITC channel (Fig. 4I) resulted in no detectable BrdU incorporation in the mural trophectoderm, but normal levels of incorporation in the ICM and polar trophectoderm (Fig. 4J; $n=55)$. Comparable frequencies of outgrowth $(>95 \%)$ were observed for control blastocysts (47/50) and JC-1stained blastocysts illuminated in the FITC channel (40/42; e.g. Fig. 4G).

In the first study of embryos illuminated in the RITC channel, 40\% (18/44) initiated outgrowth during the first $24-36 \mathrm{~h}$ of culture and when observed after $48 \mathrm{~h}$, the trophectodermal component contained few cells (Fig. 4K), 

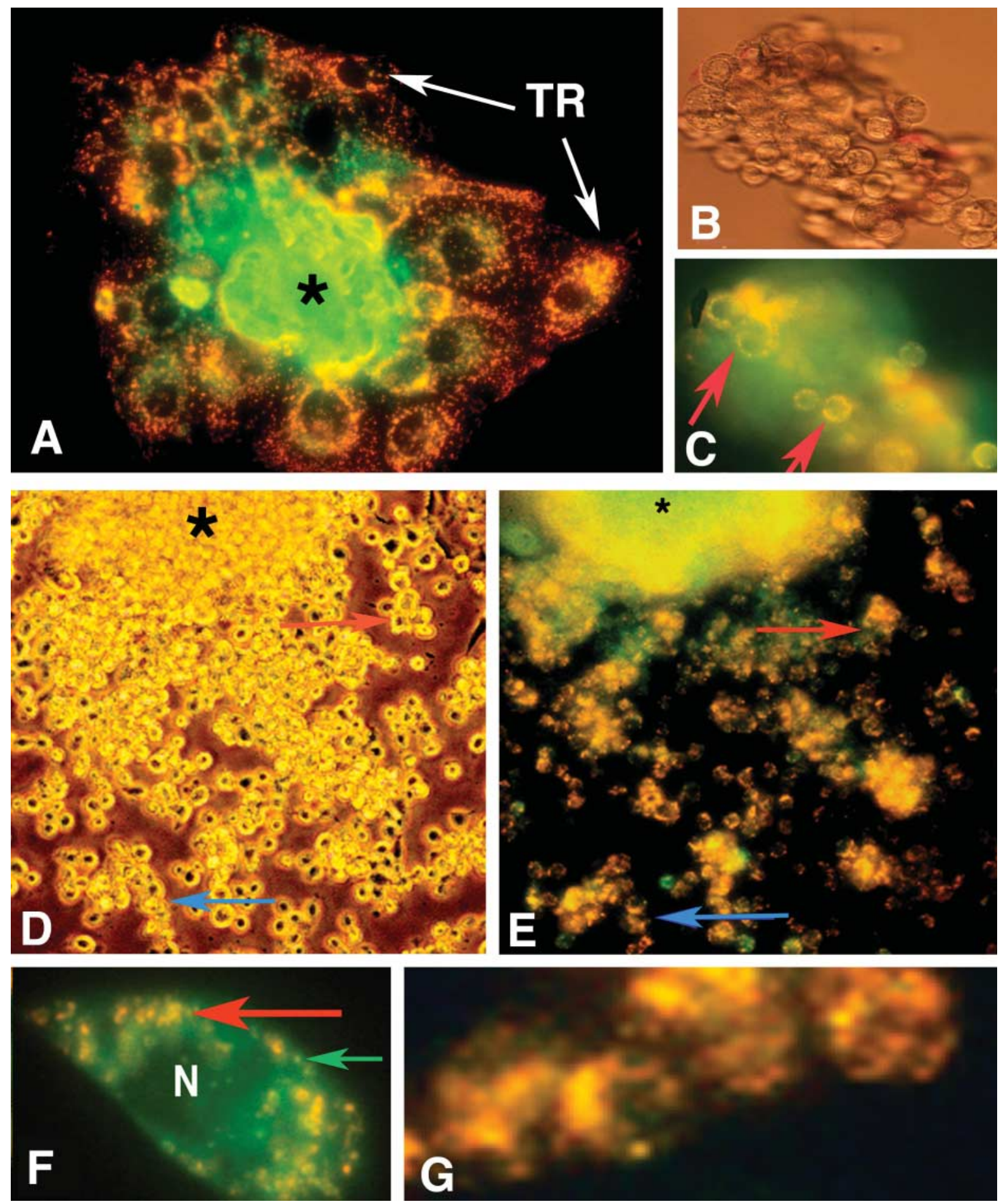

Figure 3 Differences in mitochondrial polarity in cells derived from the ICM component of blastocyst outgrowths are shown in these fluorescent images of JC-1-stained cells. (Panel A) Typical distribution of cells containing high- (trophectoderm, TR) and low-polarized (ICM indicated by asterisk) mitochondria in an undisturbed outgrowth from a normal day-4.5 embryo after 1.5 days of culture. (Panel B) Representative example of an ICM core from a nascent outgrowth that was mechanically dissected from the outgrowth (asterisk, panel A), and stained with JC-1 during dissociation (panel B). Peripheral cells showing apical J-aggregate fluorescence are indicated by red arrows in panel C. (Panel D) Four-day-old culture of pooled ICM cores composed entirely of cells that are relatively small, uniform in size and mononucleated. Differences in mitochondrial polarity are cell-contact dependent with those in dense regions containing low-polarized organelles (asterisk, panels D and E). For cells with free margins, the corresponding mitochondrial polarity was either high (blue arrow, panel $\mathrm{E}$, and at higher magnification in panel G) or a mixture of high-and low-polarized organelles (red arrow, panel E, and at higher magnification in panel F). The apparent balance between highand low-polarized mitochondria in the less dense regions of the ICM-derived cultures was related to the extent of intercellular association. 

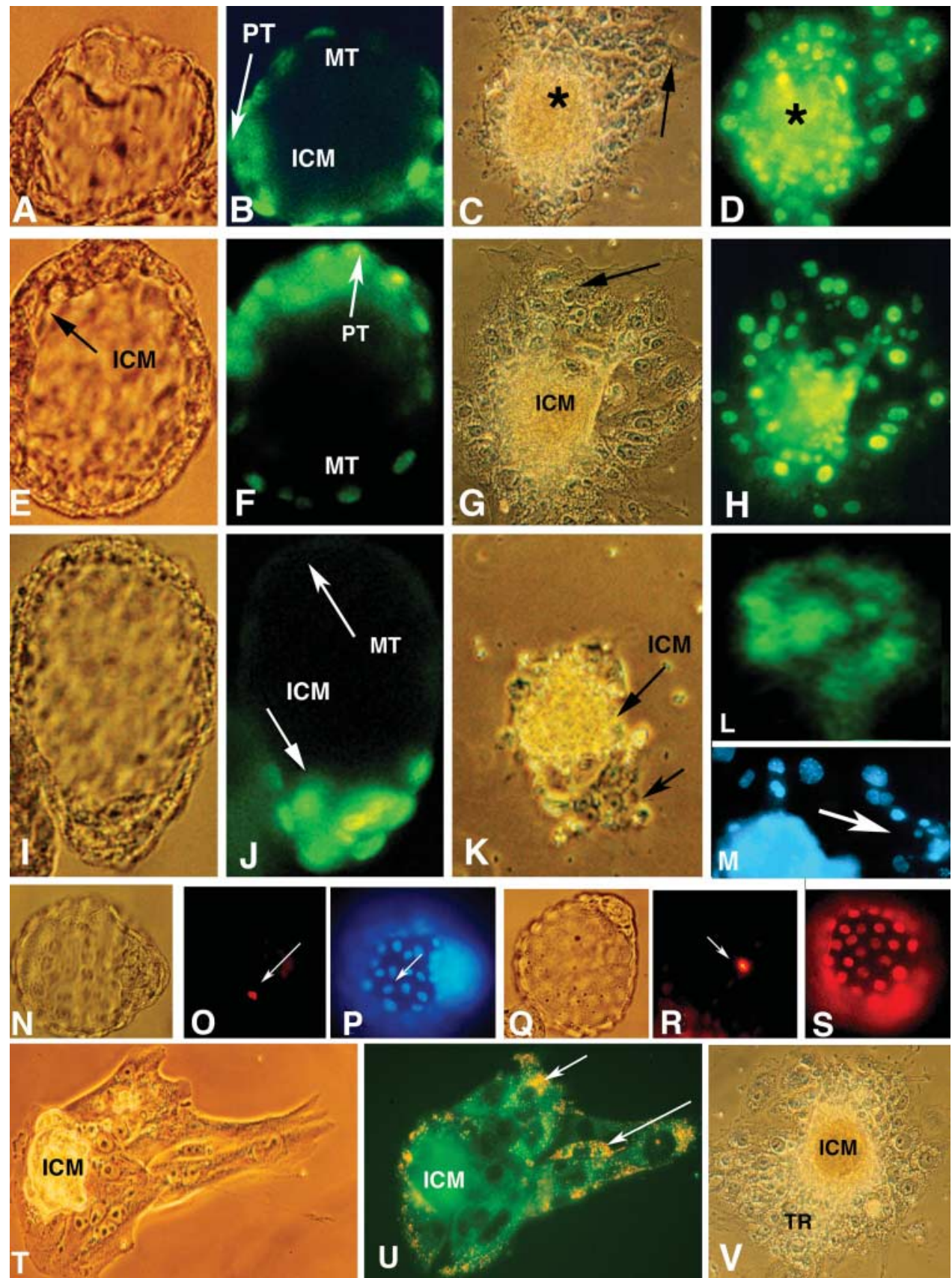

Figure 4 The typical pattern of BrdU incorporation into the ICM, mural (MT) and polar (PT) trophectoderm in a normal (panel A) day-4.5 mouse blastocyst, and a representative example of incorporation into a typical nascent blastocyst outgrowth (panel D), are shown in these light and fluorescent microscopic images. The ICM is indicated by an asterisk and trophectodermal cells by an arrow. Panels $\mathrm{E}-\mathrm{M}$ show the effects of photosensitization on BrdU incorporation in JC-1-stained, day-4.5 blastocysts (panels E and I), and representative outgrowths from similar embryos (panels G and K) after illumination in the FITC (panels F and H) or RITC (panels J and L) channels (see text for details). The arrow in panel M shows DAPI-stained nuclei in small mononucleated cells that emerged from outgrowths after several days of culture of JC-1-stained blastocysts illuminated in the RITC channel. Panel O shows a single, TUNEL-positive nucleus in a normal day-4.5 blastocyst (panel N), and its apparent location within the embryo after DAPI staining is indicated by an arrow in panel P. panel Q is an RITC-illuminated, JC-1-stained blastocyst that remained intact for 4 days after exposure. A single fluorescent nucleus indicating guanosine oxidation was detected after staining with Texas Red Avidin. All nuclei were fluorescent after exposure to peroxide (panel S). In comparison to RITC-channel-exposed, JC-1-stained blastocysts that initiated outgrowth on day 1 after exposure (e.g. panel K), similar embryos that remained intact for several days (e.g. panel Q) showed a relatively normal pattern of outgrowth on day 1 (panel T), although the intensity and distribution of J-aggregate-positive cells was reduced when compared with untreated embryos (e.g. Figures $1 \mathrm{~S}$ and $2 \mathrm{~A}$ ). However, by day 2, the appearance of the cultures was characteristic of blastocyst outgrowths (panel V). 
and $\mathrm{BrdU}$ incorporation was confined to the ICM core (Fig. 4L). However, small, mononucleated cells (arrow, Fig. 4K) first appeared around the ICM core on OG day 3 (DAPI-stained nuclei, white arrow, Fig. 4M) and, by light microscopy, were phenotypically comparable to cells derived from the disaggregated ICM cores and their progeny, as described above (Fig. 3D). A second series of exposures was undertaken to confirm these findings with a larger number of embryos; approximately 60\% (59/105) remained intact and unattached for as many as 5 days of culture (e.g. day 4, Fig. 4Q), and, during this time, showed no apparent increase in trophectodermal or ICM cell numbers (nuclear staining comparable to Fig. 1G). However, approximately $60 \%(36 / 59)$ of these embryos initiated outgrowth at days 3-4 of culture (e.g. Fig. 4T, OG day 1), but, unlike their siblings, which outgrew earlier (e.g. OG day 1, Fig. 4K), trophectodermal cells increased in number during time-lapse-monitored culture (OG day 2, Fig. 4V), and the pattern of $\mathrm{BrdU}$ staining was comparable to those observed days earlier in outgrowths from their FITC-channel-exposed siblings (comparable to Fig. 4H). Like early outgrowths from untreated blastocysts, outgrowths from blastocysts illuminated in the RITC channel showed J-aggregate fluorescence in trophectodermal, but not ICM, cells (Fig. 4U). Initially, the J-aggregate signal was punctate and confined to apical regions of outgrowing cells. However, with continued outgrowth (e.g. Fig. 4V), the intensity and distribution of J-aggregate fluorescence were comparable to their normal counterparts (e.g. the pattern shown in Fig. 2B). About 40\% (23/59) of these blastocysts failed to outgrow after 5 days of time-lapse-monitored culture (see below).

\section{Analysis of DNA fragmentation and oxidation}

As noted previously, photosensitization with mitochondria-specific fluorescent probes has been associated with the production of oxidative free radicals that are thought to induce nuclear DNA fragmentation (detected by TUNEL staining) and eventual apoptotic cell death. To determine whether exposure of JC-1-stained blastocysts in the RITC channel has similar effects, TUNEL and Texas Red Avidin staining were performed to detect DNA fragmentation and guanosine oxidation respectively. Approximately $30 \% \quad(11 / 37)$ of day-4.5 blastocysts (Fig. 4N) showed one or two TUNEL-positive nuclei (arrow, Fig. 4O), but avidin fluorescence indicative of guanosine oxidation was not detected (image not shown). Figure 4P shows nuclear fluorescence after DAPI staining of the TUNEL-positive embryo in Fig. 4O. For JC-1-stained embryos that remained dormant (i.e. unattached or expanded) for 3-5 days after illumination in the RITC channel (e.g. day 4, Fig. 4Q), the occurrence of TUNELpositive nuclei was the same as for the controls, with only an occasional positive nucleus observed in about $30 \%(12 / 38)$ of the blastocysts examined (the rest were TUNEL-negative). When stained with Texas Red Avidin on culture days 3-5, one or two positive nuclei were detected in under $10 \%(2 / 29)$ of the expanded blastocysts (e.g. day 4 of culture, Fig. 4Q and R). After brief treatment with hydrogen peroxide, positive fluorescence was observed in all nuclei of control blastocysts $(n=17)$ and in all blastocysts that remained unattached and expanded after illumination in the RITC channel (Fig. 4S, day 4 of culture, $n=21$ ).

\section{Analysis of ATP contents in normal and delayed implanted blastocysts}

The average net ATP content $( \pm 22)$ of hatched blastocysts on day 4.5 of pregnancy $(n=106)$ was $127 \mathrm{fmol}$, and 187 and $196 \mathrm{fmol}$ respectively on days $3(n=61)$ and 5 $(n=61)$ of diapause. The differences in ATP content between normal and delayed implanted embryos were significant $(P=0.01)$. The ATP content of JC-1-stained blastocysts illuminated in the FITC $(n=81)$ and RITC channels $(n=81)$ was measured after $20-24 \mathrm{~h}$ of culture. The average ATP content was $131 \mathrm{fmol}$ (FITC, \pm 20 ) and $90 \mathrm{fmol}$ (RITC, \pm 20 ) respectively. The average ATP content of blastocysts illuminated in the RITC channel that did not outgrow after $2(n=33), 3(n=26), 4(n=29)$ or $5(n=19)$ days of culture was $85 \mathrm{fmol}( \pm 18)$, and this lower level was significant $(P=<0.01)$ with respect to untreated blastocysts and JC-1-stained embryos illuminated in the FITC channel. In contrast, the net ATP content of RITC-channel-exposed embryos that showed indications of incipient outgrowth (focal elaboration of filapodia and bulbous projections; see below) after 3 $(n=25)$ or $4(n=21)$ days of time-lapse-monitored culture was $138( \pm 21)$, a level comparable to the one measured in normal day-4.5 embryos.

\section{Time-lapse analysis}

The behavior of hatched blastocysts recovered from the uterus on day $4.5(n=25)$ of pregnancy was investigated by time-lapse microscopy. After $8-10 \mathrm{~h}$ of culture (Fig. 5A), small (asterisk), highly motile filopodial projections (arrow) emerged from the abembryonic region of the mural trophectoderm. Expanded diapausing blastocysts $(n=31)$ cultured immediately after removal from the uterus on days $3(n=20)$ or $5(n=16)$ of delayed implantation showed no obvious morphodynamic activity during the first $5 \mathrm{~h}$ in vitro (Fig. 5B). However, around $6 \mathrm{~h}$, filopodia (arrow, Fig. 5C) emerged from the abembryonic portion of the mural trophoblast. The dynamic nature of these projections was indicated in time lapse by cycles of evolution and resorption that increased in frequency by $16 \mathrm{~h}$ in control blastocysts, and by $12 \mathrm{~h}$ of culture in blastocysts released from diapause in vitro (arrow, Fig. 5D). In all instances, focal filopodial formation preceded the establishment of robust outgrowths, and in some time-lapse sequences, filopodia were observed to detach from the embryo and 'crawl' on the glass culture surface for several hours (see below). 

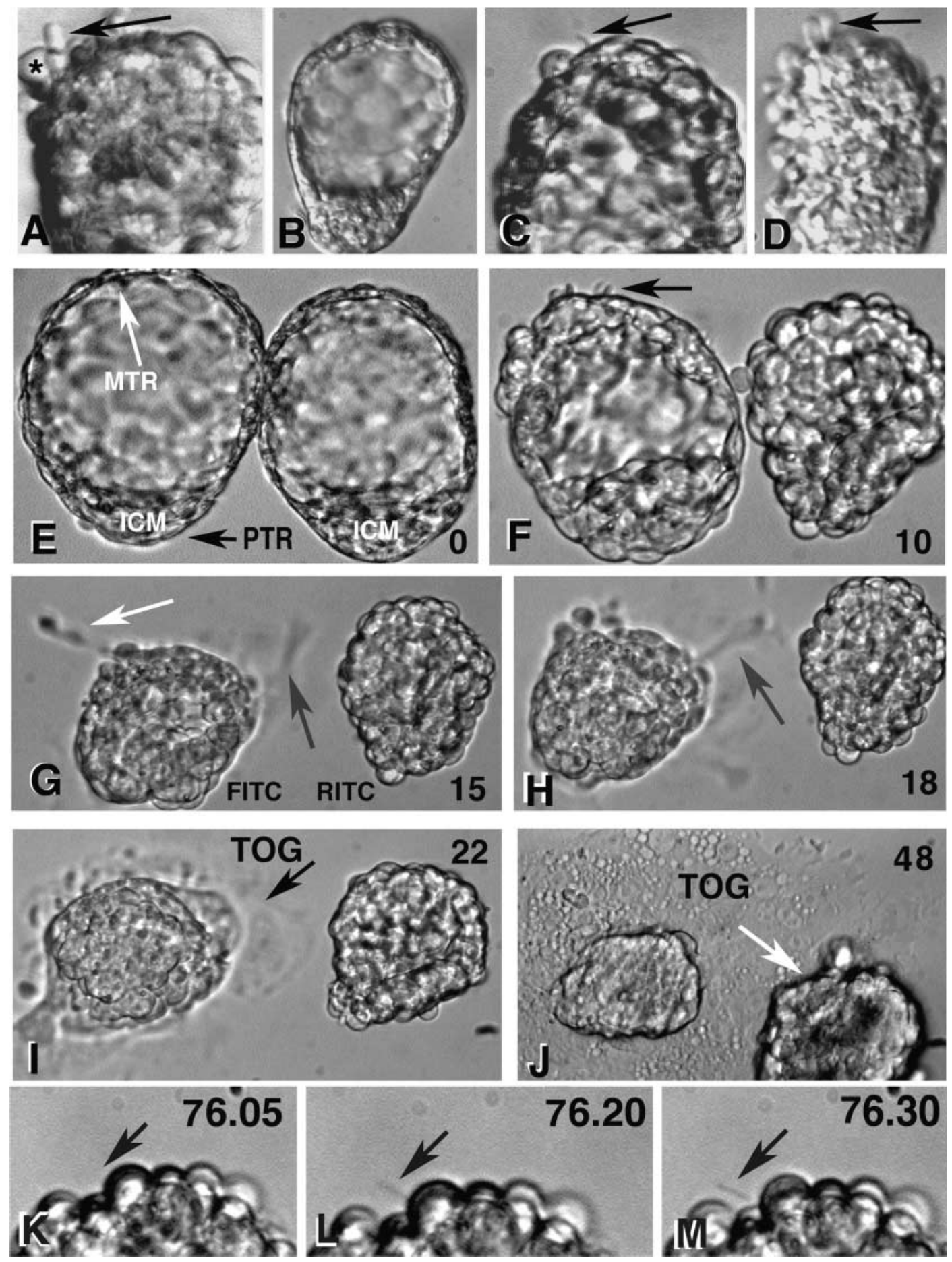

Figure 5 Static images of morphodynamic processes taken from time-lapse monitoring of normal day-4.5. blastocysts (panel A), blastocysts reactivated from diapause in vitro (panels B-D), and JC-1-stained blastocysts photosensitized in the FITC (left) and RITC channels (right) (panels $\mathrm{E}$ and F) are shown. For normal embryos, the emergence of bulbous projections (asterisk, panel A) and filopodia (arrow, panels A, F and G) from the abembryonic portion of the mural trophectoderm preceded robust trophectodermal outgrowth and expansion (TOG, panels I and J). Their appearance in reactivated embryos (panel B) occurred some hours later than in normally progressing embryos. Spatial and temporal aspects of their occurrence in FITC-channel-illuminated embryos (arrows, right-hand embryo, panel F) were normal. In contrast, for RITC-channel exposed, JC-1-stained blastocysts, they often appeared after 2 or more days in culture (arrow, panel J). In these instances, filopodia appeared suddenly (e.g. from $76 \mathrm{~h} 5 \mathrm{~min}$ to $76 \mathrm{~h} 20 \mathrm{~min}$ of culture; panels $\mathrm{K}$ and L). Some filopodia detached (panel M) and were observed to 'crawl' around the surface of the culture dish for several hours. As with untreated embryos, the elaboration of numerous filopodia preceded outgrowth for these embryos. The time of observation (hours) in panels E-J is indicated in the upper or lower right-hand portion of each image. 
For time-lapse studies of photosensitized blastocysts, embryos obtained from the same animal were cultured in pairs, with the FITC- and RITC-exposed embryos placed on the left and right respectively (Fig. 5E-J). Morphodynamic activities of blastocysts illuminated in the FITC were temporally and spatially identical to their unexposed control and reactivated counterparts, and exhibited robust outgrowth, as shown in Fig. 5G-J, which are representative images of the first $48 \mathrm{~h}$ of culture. The arrow in Fig. 5F denotes filopodia that had developed about $2 \mathrm{~h}$ earlier in the abembryonic trophectoderm. In contrast, most blastocysts illuminated in the RITC channel showed no apparent morphodynamically activity during 3-5 days of culture $(n=38)$, and while some remained expanded, over $80 \%$ (31/38) were in a persistent state of 'collapse', which initially occurred with a spontaneous discharge of blastocelic fluid around h 10 of culture (Fig. 5F-J).

In comparison to control blastocysts or embryos reactivated from diapause in vitro, outgrowth for most JC-1stained blastocysts illuminated in the RITC channel began between days 3 and 5, including those cocultured with siblings illuminated in the FITC channel (see above). Outgrowth was also preceded by the elaboration of filopodia (arrows, Fig. 5K-M), which appeared rather suddenly in embryos that seemed morphodynamically 'inert' prior to onset of this activity. In Fig. 5J, the region of the trophectoderm from which filopodia emerged is indicated by an arrow. It is assumed that the abembryonic portion of the mural trophoblast was involved owing to the absence of embryo movement during time-lapse-monitored culture. As described previously, trophectodermal outgrowths from embryos illuminated in the RITC channel were generally less robust if they occurred within the first day or two of culture, but were largely typical of this process if initiated on or after culture day 3 .

\section{Discussion}

The primary role of mitochondria in the preimplantationstage embryo has traditionally been considered a metabolic one, that is, to provide ATP by means of oxidative phosphorylation, and adverse effects of numerical or structural defects that occur naturally (Müller-Höcker et al. 1996, Van Blerkom et al. 2000) or are experimentally induced (Eichenlaub-Ritter et al. 2004, Thouas et al. 2004; reviewed by Cummins 2002, Brenner 2004) are usually considered in this context. Mitochondrial polarity is a physiochemical property that results from the outward pumping of protons across the inner mitochondrial membrane, creating a proton gradient with two components, an inner membrane potential $(\Delta \Psi \mathrm{m})$ and a $\mathrm{pH}$ gradient; the energy stored in either component drives the conversion of ADP to ATP by respiratory chain enzymes. Differences in the relative magnitude of the membrane potential detected with $\Delta \Psi \mathrm{m}$-specific probes have been suggested to reflect corresponding differences in mitochondrial activity or function that affect the competence of mature oocytes and early embryos in the mouse and man (Wilding et al. 2001, 2002, 2003, Van Blerkom et al. 2002, Acton et al. 2004, Jones et al. 2004, Van Blerkom 2004). For these studies, JC-1 has been the probe of choice owing to the specificity with which it reports high and low $\Delta \Psi \mathrm{m}$ in living cells (Salvioli et al. 1977). Comparatively low-polarized mitochondria (under $\sim 140 \mathrm{mV}$ ) fluoresce green, reflecting accumulation of the JC-1 monomer in the mitochondrial matrix, while comparatively high-polarized mitochondria (over $\sim 140 \mathrm{mV}$ ) fluoresce orange to red, as a result of monomer multimerization into so-called J-aggregates that shift in the emission spectrum to longer wavelengths (for review of JC-1, see Reers et al. 1995).

Differences in the spatial distribution of high- and lowpolarized mitochondria in mouse and human oocytes and cleavage-stage embryos have been suggested to represent discrete regions of differential mitochondrial activity with distinct or focal regulatory functions (e.g. ATP production, calcium regulation, post-translation modification; Van Blerkom et al. 2002, Van Blerkom 2004). For blastocyststage embryos, Van Blerkom et al. (2002) detected highpolarized mitochondria in the trophectoderm, but not the ICM, and proposed that different states of mitochondrial polarization may reflect corresponding differences in organelle activity or function in the two lineage-determining (progenitor) cell types of the mammalian embryo. Here, the physiologic and possible developmental significance of $\Delta \Psi \mathrm{m}$ during the terminal stage of the preimplantation period was investigated further.

\section{$\Delta \Psi m$ in normal mouse blastocysts}

The present results confirm and extend our previous findings (Van Blerkom et al. 2002) by demonstrating that the occurrence and intensity of high- and low-polarized mitochondria are location- and cell-type specific in the hatched mouse blastocyst as follows:

1. the ICM was consistently J-aggregate negative, indicating that the corresponding mitochondria are low polarized

2. polar trophectodermal cells overlying the ICM exhibited no detectable J-aggregate fluorescence or an intracellular signal that was scant, indicating that high-polarized mitochondria are largely absent in this region

3. the mural trophectoderm exhibited regional differences in fluorescent intensity that extended from the abembryonic (high) to the peripolar regions (low).

The specificity of JC-1 labeling was confirmed by the absence of J-aggregate fluorescence after treatment with the $\Delta \Psi \mathrm{m}$-collapsing proton ionophore $\mathrm{FCCP}$, and by the detection in mural trophectodermal cells of J-aggregatepositive, rod-shaped elements that were equivalent in size and distribution to mitochondria identified with other organelle-specific fluorescent probes. Comparable 
patterns, distributions and intensities of J-aggregate fluorescence detected under different staining conditions and after mechanical manipulations of embryos support our previous contention (Van Blerkom et al. 2002) that JC-1 reports actual location- and cell-type-specific differences in embryo mitochondrial polarity. However, the possibility that the magnitude of $\Delta \Psi \mathrm{m}$ is related to mitochondrial fine structure needs to be considered, because, unlike mitochondria, in the oocyte and early cleavage-stage embryo (Van Blerkom et al. 2002), differences in matrix density, the number of lamellar cristae, and the degree of organelle elongation distinguish ICM and polar trophectodermal mitochondria from their mural trophectodermal counterparts at the late blastocyst stage (Van Blerkom \& Motta 1979, Van Blerkom et al. 1979). At present, these differences are not thought to be related to the magnitude of $\Delta \Psi \mathrm{m}$, because the undeveloped pericortical mitochondria in the oocyte and early cleavage-stage embryo, which are small, spherical organelles with a few, short cristae that surround, but rarely penetrate, a matrix of very highdensity, are J-aggregate positive (Van Blerkom et al. 2002). The occurrence of J-aggregate-positive cells in ICM outgrowths (see below) is a further indication that low $\Delta \Psi \mathrm{m}$ is not an intrinsic property of their mitochondria.

\section{$\Delta \Psi \mathrm{m}$ in delayed implanted mouse blastocysts}

Aerobic metabolism with glucose as the primary metabolic substrate is the major pathway of energy production in the mouse blastocyst, and respiration from fully developed mitochondria accounts for over $85 \%$ of all ATP produced (Benos \& Balaban 1983, Trimarchi et al. 2000). The facultative delayed implantation mouse model was used to study the relationship between ATP production and $\Delta \Psi \mathrm{m}$ because mitochondria are metabolically suppressed during the diapause. The decreased energy requirement for diapausing mouse blastocysts results from significantly reduced levels of transcription, translation and protein phosphorylation, and the cessation of DNA replication and cell division (Van Blerkom et al. 1979, Weitlauf \& Kiessling 1980, Hamatani et al. 2004, Lopes et al. 2004). Nieder and Weitlauf (1984) showed that diminished mitochondrial respiratory activity in diapausing mouse blastocysts is associated with significant elevation in cytoplasmic ATP content, and proposed that a constitutively high ATP content caused allosteric inhibition of phosphofructokinase and coincident reduction in glucose oxidation. Increased mitochondrial metabolism detected some hours after blastocyst reactivation could be a consequence of the reversal of this inhibition for the existing enzyme combined with translation from nascent phosphofructokinase transcripts (Hamatani et al. 2004). Our finding of elevated levels of ATP in diapausing blastocysts supports the notion of allosteric inhibition advanced by Nieder and Weitlauf (1984).

Changes in $\Delta \Psi \mathrm{m}$ (high to low) have been reported for early mouse and human embryos that develop abnormally or arrest prematurely in vitro (Wilding et al. 2002, 2003, Acton et al. 2004), are developmentally compromised after photosensitization of mitochondria stained with $\mathrm{r} 123$ (Thouas et al. 2004), or undergo iatrogenically induced reductions in polarity under certain culture conditions (Van Blerkom et al. 2003) or after cryopreservation (Ahn et al. 2002, Jones et al. 2004). These changes are largely considered in the context of a coincident reduction in mitochondrial metabolic activity, and for diapausing embryos, we had anticipated a downward shift in $\Delta \Psi \mathrm{m}$ owing to the suppression of mitochondrial respiratory activity. However, during the 7 days of delayed implantation examined in the present study, the location, cell-type specificity and relative intensity of J-aggregate fluorescence remained unchanged in embryos with elevated cytoplasmic ATP contents. A rapid upregulation of mitochondrial metabolic activity during JC-1 staining is an unlikely explanation for this finding because the normal pattern of J-aggregate fluorescence was detected within the first 5 min of staining in medium devoid of metabolic substrates. The translation of functional respiratory enzymes from nascent transcripts seems equally unlikely to occur during this time (Hamatani et al. 2004); we suggest that the detection of J-aggregate fluorescence reflects the in vivo state and that high polarity in mural trophectodermal mitochondria is maintained during the diapause. Because ATP is required for a potential difference to exist across the inner mitochondrial membrane, the relatively high ATP content of diapausing mouse blastocysts may be sufficient simultaneously to suppress mitochondrial metabolism and support high $\Delta \Psi \mathrm{m}$. A similar situation exists in $\rho^{\circ}$ cells, whose mitochondria have neither DNA nor a functional respiratory chain cells, yet consume about $13 \%$ of the ATP produced by glycolysis to maintain a $\Delta \Psi \mathrm{m}$ of $\sim 110 \mathrm{mV}$ (Appleby et al. 1999). Possible reasons why high polarity is retained are discussed below.

\section{$\Delta \Psi \mathrm{m}$ and the regulation of development during diapause}

Our results raise several questions concerning how mitochondria may be involved in the regulation of development during the peri-implantation period; in particular, whether high-polarized mitochondria have specialized functions in this regard, especially during the diapause. One possibility under investigation is related to mitochondrial regulation of calcium homeostasis. Calcium is a ubiquitous regulator of numerous cell functions, including control of the cell cycle, cytoskeletal/cytoplasmic remodeling, levels of mitochondrial respiration, morphodynamic movements, and, through various signal transduction pathways, differential gene expression and cell death (Duchen 2000, Rutter \& Rizzuto 2000, Berridge et al. 2003, Webb \& Miller 2003). All of these processes are either downregulated or arrested during the diapause (Van Blerkom et al. 1979, Hamatani et al. 2004, Lopes et al. 2004). Also well known is the 
involvement of mitochondria in general (Pozzan et al. 2000), and high-polarized mitochondria in particular (Loew et al. 1994, Babcock et al. 1997, Hofer \& Brown 2003), in the regulation of intracellular free calcium in differentiated cells, and a similar role has been suggested for mitochondria in mouse and human oocytes and early embryos (Liu et al. 2001, Van Blerkom et al. 2002, Dumollard et al. 2003, 2004, Jones et al. 2004, Makabe \& Van Blerkom 2006).

To test this possibility, we measured in preliminary studies changes in levels of cytoplasmic free calcium after mitochondrial depolarization was chemically induced in normal day-4.5 embryos and on day 5 of delayed implantation (unpublished). Embryos were preloaded with the fluorescent calcium reporter Fluo-4 AM in medium free of calcium, magnesium and metabolic substrates (Van Blerkom et al. 2003, Jones et al. 2004), and then exposed to the proton ionophore FCCP at concentrations shown to dissipate $\Delta \Psi \mathrm{m}$ abruptly and induce a significant increase of intracellular free calcium in mature mouse oocytes (Van Blerkom et al. 2002, 2003). Changes in cytoplasmic free calcium levels were determined quantitatively by measuring the increase in relative fluorescence intensity (above the pre-FCCP baseline) by scanning laser confocal microscopy. After mitochondrial depolarization, the increase in free calcium in diapausing embryos was 3-4 times higher than in their normal counterparts. If these initial findings are confirmed, it could suggest that high polarity is maintained during the diapause in order to sequester calcium in mitochondria such that cytoplasmic levels are reduced, but can return to 'normal' (baseline) when delayed implantation ends. Although speculative, mitochondrial calcium sequestration and release in diapausing/reactivated embryos may be associated with the down- and upregulation respectively of regulatory proteins whose function or activity is calcium dependent (Hamatani et al. 2004). In this regard, it will be of interest to determine whether changes in free calcium occur after reactivation and are temporally correlated with the upregulation or reinitiation of biosynthetic, cell-cycle (Van Blerkom 1979, Weitlauf \& Kiessling 1980, Lopes et al. 2004) and morphodynamic activities (see below).

\section{Differential effects of mitochondrial photosensitization}

Photosensitization of mitochondria stained with organellespecific fluorescent dyes has been widely used to study the cellular and developmental consequences of 1 . perturbing electron transport and reducing metabolic activity, and 2. inducing the generation of reactive oxidative species at levels that cause structural damage to mitochondria and DNA, or the release of proapoptotic factors. For these studies, preloading with a fluorescent probe is usually followed by an acute exposure to highintensity ultraviolet illumination at appropriate excitation and emission frequencies, for times sufficient to have detectable pathophysiologic effects. When the developmental sequelae are considered in the context of the central role mitochondria have in cell function, it is not surprising that the downstream effects of altered organelle function in oocytes and early embryos (Lane \& Gardner 2005) induced by photosensitization (Thouas et al. 2004) are largely dose-dependent and often developmentally lethal.

Here, we used photosensitization to derive a sublethal exposure for JC-1-stained blastocysts to determine whether cells containing high- or low-polarized mitochondria are differentially affected. Illumination in the FITC channel had no discernible effects, while exposure in the RITC channel reduced the net ATP content, inhibited BrdU incorporation in the (high-polarized) mural trophectoderm, and delayed or precluded outgrowth. However, outgrowth-arrested embryos showed no increase in the frequency of nuclei exhibiting fragmented DNA or indications of guanosine oxidation, indicating that this protocol did not induce apoptosis or other forms of lethal cytopathology. We suggest that putative fluorescence resonance of the J-aggregate multimers in high-polarized mitochondria may cause an acute, but not irreversible reduction in mitochondrial activity that appears to include ATP generation. Whether the magnitude of $\Delta \Psi \mathrm{m}$ was affected in high-polarized mitochondria could not be determined under the present conditions, because, once formed, J-aggregates are metastable structures that continue to fluoresce, albeit at reduced intensity, even if $\Delta \Psi \mathrm{m}$ is dissipated by FCCP or fixation (Van Blerkom et al. 2002). Likewise, it is unknown whether the arrest of cell division in the ICM of embryos in outgrowth-delay for several days indicates a direct effect of photosensitization on low-polarized mitochondria. However, photosensitization does seem to induce a developmental 'quiescence' with cellular and phenotypic similarities to the natural diapause. Whether the reduced cytoplasmic ATP content measured during delayed outgrowth is sufficient to downregulate critical cellular activities (such as DNA synthesis and cytokinesis) that could induce a diapause-like state remains to be determined.

Our studies show that mitochondria have a central regulatory function during the peri-implantation stage that mediates the ability of mouse embryo to develop progressively. Cellular domains containing high-polarized mitochondria may be particularly important in this regard owing to their spatial association with morphodynamic processes that precede implantation. For example, the initial stages of implantation in the mouse involve dynamic activities localized to abembryonic mural trophectoderm that are characterized by the elaboration of focal clusters of filopodia and bulbous protrusions. The filopodia are thought to establish the first stable contacts with the endometrial epithelium, and they later physically participate in the implantation process (Potts 1968, Bergstrom \& Nilsson 1976). Trophoblast giant cells, which become a terminally differentiated, nondividing (polyploid) cluster of cells involved in the invasive phase of 
implantation (Maris et al. 1988), are first identified in the day-5 mouse embryo as 'bulbous projections' from the abembryonic trophectoderm (Kaufman 1983). Here, timelapse analysis showed the elaboration of filopodia and bulbous projections from this region in normal (on day 5 in vitro), reactivated, and FITC-channel-exposed, JC-1stained blastocysts. In all instances, active filopodial formation preceded robust trophectodermal outgrowth. In contrast, most JC-1-stained blastocysts illuminated in the RITC channel showed no apparent morphodynamic activity after several days of coculture with normally outgrowing siblings that had been illuminated in the FITC channel. However, when 'delayed' outgrowth was initiated, it, too, was always preceded by the appearance of filopodia and bulbous projections. Owing to the retention of residual J-aggregate fluorescence, we could not determine quantitatively whether this morphodynamic activity is preceded by a focal change in $\Delta \Psi \mathrm{m}$. For embryos in which comparatively normal outgrowth occurred after a delay of several day, the results indicate that DNA replication, cell division, filopodial formation and outgrowth may await the restoration of 'normal' mitochondrial function. This interpretation was supported by cytoplasmic ATP contents at levels comparable to day 4.5 in these embryos, where the appearance of filopodia indicated outgrowth was imminent. Restoration of mitochondrial activity or function to near normal levels may be especially relevant to the expression of morphodynamic activities in the abembryonic region, which typically displays higher relative intensities of J-aggregate fluorescence.

\section{Cell-type-specific $\Delta \Psi \mathrm{\Psi m}$ in the peri-implantation blastocyst may be determined by extrinsic factors}

Our previous studies suggest that within oocytes and blastomeres, spatial differences in mitochondrial polarity are cell-contact associated rather than a reflection of differential metabolic metabolism (Van Blerkom et al. 2002, 2003). The present results indicate that a similar situation may exist at the blastocyst stage. Trimarchi et al. (2000) found oxygen consumption to be uniform along the entire circumference of the hatched mouse blastocyst, and concluded that regional differences in levels of oxidative metabolism probably do not exist. If this accurately reflects underlying metabolic activity, cellular domains of high and low $\Delta \Psi \mathrm{m}$ would appear to be unrelated to corresponding differences in mitochondrial oxidative phosphorylation, and this appears to be the case during the diapause as well.

A nonmetabolic origin for differential polarity in the peri-implantation mouse embryo is suggested by the finding that within cells, high or low polarity is related to the extent and nature of intercellular contact and communication. Diaz et al. (1999) showed for several differentiated cell lines that high-polarized (J-aggregate-positive) mitochondria occurred in the subplasmalemmal cytoplasm at the free margins of cells, whereas low-polarized (J-aggregate-negative) mitochondria occurred in the interior of the cytoplasm and in subplasmalemmal zones where intercellular contacts existed. The state of polarity was found to be a dynamic one in which the loss of intercellular contact was associated with a shift from low to high $\Delta \Psi$, while the opposite occurred when such contacts were established. Because the relative magnitude of $\Delta \Psi \mathrm{m}$ in subplasmalemmal mitochondria can be influenced by ionic and electrical fluxes within and between cells (Ichas et al. 1997, Diaz et al. 1999), the occurrence of highpolarized mitochondria at free margins may reflect a need to 'work harder' to regulate calcium homeostasis than their counterparts deeper within the cytoplasm or where cells membranes are in direct contact and ionic homeostasis is facilitated by specialized (gap) junctions (Diaz et al. 1999).

A similar relationship with mitochondrial polarity has been proposed for the oocyte and cleavage-stage embryo (Van Blerkom et al. 2003, Van Blerkom 2004); high-polarized mitochondria occur at the free margins and lowpolarized mitochondria are located internally; however, for the cleavage-stage embryo, low-polarized mitochondria also occur in the subplasmalemmal cytoplasm subjacent to zones of contact (Van Blerkom et al. 2002), where intercellular communication involves gap junctions (Van Blerkom \& Motta 1979). The dynamic nature of this spatial pattern was demonstrated by disaggregating twoand four-cell mouse embryos and repositioning and realigning blastomeres such that formerly J-aggregate-negative cortical domains became positive, and vice versa (Van Blerkom et al. 2002).

The same types of intercellular interactions that influence the magnitude of $\Delta \Psi \mathrm{m}$ in somatic cell cultures exist in the hatched blastocyst. For example, the elongated apical and basal surfaces of the abembryonic mural trophectoderm are free, but toward the peripolar region, focal contacts with early migrating endodermal cells are established (Van Blerkom et al. 1979). The basal surface of the polar trophectoderm fully associates with the ICM, whose cells are largely in close contact throughout their margins (Van Blerkom \& Motta 1979, Makabe \& Van Blerkom 2006). The occurrence and relative intensity of high-polarized trophectodermal mitochondria coincides with the presence or absence of subjacent cells and where present, the extent of association. Similar to the situation observed in cultured cells, gap junctions that regulate the flow of ions between cells exist between ICM cells and between the polar trophectoderm and ICM (Van Blerkom \& Motta 1979). In this respect, gap-junction functionality and density, combined with the degree of intercellular contact, may largely determine cell-type-specific $\Delta \Psi \mathrm{m}$ within the peri-implantation blastocyst. The present studies of undisturbed blastocyst outgrowths, disaggregated ICM cores and proliferating ICM-derived cells, demonstrate that low polarity is not an intrinsic property, but one related to the extent of intercellular contact. We suggest that cell- and 
location-specific mitochondrial polarity at the blastocyst stage is determined by the normal intercellular morphodynamics of cavitation and blastocele expansion. Motosugi et al. (2005) recently proposed that these same intercellular dynamics alone lead to the specification of the first developmental polarity in the mouse embryo, the establishment of the embryonic-abembryonic axis. It will be of interest to determine whether other regulatory functions of mitochondria (e.g. oxygen sensing) whose activity may be $\Delta \Psi \mathrm{m}$ associated are involved in the molecular specification of cell types, or influence their corresponding state of differentiation (trophectoderm) or totipotency (ICM) at the blastocyst stage.

\section{Acknowledgements}

The authors declare that there is no conflict of interest that would prejudice the impartiality of this scientific work.

\section{References}

Acton B, Jurisicova A, Jurisica I \& Casper R 2004 Alterations in mitochondrial membrane potential during preimplantation stages of mouse and human embryo development. Molecular Human Reproduction $123-32$.

Ahn H, Sohn I, Kwon H, Jo D, Park Y \& Min C 2002 Characteristics of the cell membrane fluidity, actin fibers, and mitochondrial dysfunctions of frozen- thawed two-cell mouse embryos. Molecular Reproduction and Development 61 466-476.

Appleby R, Porteous W, Hughes G, James A, Shannon D, Wei Y-H \& Murphy M 1999 Quantitation and origin of the mitochondrial membrane potential in human cells lacking mitochondrial DNA. European Journal of Biochemistry 262 108-116.

Babcock D, Herrington J, Goodwin P, Park Y \& Hille B 1997 Mitochondrial participation in the intracellular $\mathrm{Ca}^{2+}$ network. Journal of Cell Biology 136 833-844.

Benos D \& Balaban R 1983 Energy metabolism of preimplantation mammalian blastocysts. American Journal of Physiology 245 [Cell Physiology 14] C40-C45.

Bergstrom E \& Nilsson O 1976 Blastocyst attachment and early invasion during oestradiol-induced implantation in the mouse. Anatomy and Embryology 149 149-154.

Berridge M, Bootman M \& Roderick L 2003 Calcium signalling: dynamics, homeostasis and remodeling. Nature Reviews. Molecular Cell Biology 4 517-529.

Biggers J \& Borland R 1976 Physiological aspects of growth and development of the preimplantation mammalian embryo. Annual Reviews in Physiology 38 95-119.

Brenner C 2004 What is the role of mitochondria in embryo competence? In Essential IVF: Basic Research and Clinical Applications, pp 273-290. Eds J Van Blerkom \& L Gregory. Boston, MA: Kluwer.

Chavez D \& Van Blerkom J 1979 Persistence of RNA synthesis during facultative delayed implantation in the mouse. Developmental Biology 70 39-49.

Cossarizza A, Ceccarelli D \& Masini A 1996 Functional heterogeneity of an isolated mitochondrial population revealed by cytofluorometric analysis at the single organelle level. Experiments in Cell Research 222 84-94.

Cummins J 2002 The role of maternal mitochondria during oogenesis, fertilization and embryogenesis. Reproductive Biomedicine Online 4 176-182.

Dedov V \& Roufogalis B 1999 Organization of mitochondria in sensory neurons. FEBS Letters 456 171-174.

Diaz G, Setzu H, Zucca A, Isola R, Diana A, Murru R, Sogos V \& Gremo F 1999 Subcellular heterogeneity of mitochondrial mem- brane potential: relationship with organelle distribution and intercellular contacts in normal, hypoxic and apoptotic cells. Journal of Cell Science 112 1077-1084.

Duchen M 2000 Mitochondria and calcium: from cell signalling to cell death. Journal of Physiology 529 57-68.

Dumollard R, Hammar K, Porterfield M, Smith P, Cibert C, Rouviere C \& Sardet C 2003 Mitochondrial respiration and $\mathrm{Ca}^{2+}$ waves are linked during fertilisation and meiosis completion. Development $130683-692$.

Dumollard R, Marangos P, Fitzharris G, Swann K, Duchen M \& Carroll J 2004 Sperm-triggered $\left[\mathrm{Ca}^{2+}\right]$ oscillations and $\mathrm{Ca}^{2+}$ homeostasis in the mouse egg have an absolute requirement for mitochondrial ATP production. Development 131 3057-3067.

Eichenlaub-Ritter U, Vogt E, Yin H \& Gosden R 2004 Spindles, mitochondria and redox potential in ageing oocytes. Reproductive Biomedicine Online 8 45-58.

Hamatani T, Daikoku T, Wang H, Matsumoto H, Carter MG, Ko MS \& Dey SK 2004 Global gene expression analysis identifies molecular pathways distinguishing blastocyst dormancy and activation. PNAS 101 10326-10331.

Hofer A \& Brown E 2003 Extracellular calcium sensing and signalling. Nature Reviews. Molecular Cell Biology 4 530-538.

Ichas F, Jouaville L \& Mazat J-P 1997 Mitochondria are excitable organelles capable of generating and conveying electrical and calcium signals. Cell 89 1145-1153.

Jones A, Van Blerkom J, Davis P \& Toledo A 2004 Cryopreservation of metaphase II human oocytes effects mitochondrial membrane potential: implications for developmental competence. Human Reproduction 19 1861-1866.

Kaufman M 1983 The origin, properties and fate of trophoblast in the mouse. In Biology of the Trophoblast, pp 23-68. Eds W Lake \& A Whyte. Amsterdam: Elsevier.

Lane M \& Gardner D 2005 Understanding cellular disruptions during early embryo development that perturb viability and fetal development. Reproduction, Fertility, and Development 17 371-378.

Liu L, Hammar K, Smith P, Inoue S \& Keefe D 2001 Mitochondrial modulation of calcium signaling at the initiation of development. Cell Calcium $30423-433$.

Loew L, Carrington W, Tuft R \& Fay F 1994 Physiological cytosolic $\mathrm{Ca}^{2+}$ transients evoke concurrent mitochondrial depolarization. PNAS 91 12579-12583.

Lopes F, Desmarais J \& Murphy B 2004 Embryonic diapause and its regulation. Reproduction 128 669-678.

Makabe S \& Van Blerkom J 2006 In Atlas of Human Female Reproductive Function: Ovarian Development to Early Embryogenesis After in Vitro Fertilization, Ch 4. London: Taylor, Francis.

MacLean-Hunter S \& Evans M 1999 Non-surgical method for the induction of delayed implantation and recovery of viable blastocysts in rats and mice by the use of tamoxifen and Depo-Provera. Molecular Reproduction and Development 52 29-32.

Maris E, Bevilacqua A \& Abrahamsohn P 1988 Ultrastructure of trophoblast giant cell transformation during the invasive stage of implantation in the mouse embryo. Journal of Morphology 198 341-351.

Motosugi N, Bauer T, Polanski Z, Solter D \& Hiiragi T 2005 Polarity of the mouse embryo is established at blastocyst and is not prepatterned. Genes and Development 19 1081-1092.

Müller-Höcker J, Schäfer S, Weis S, Münscher Ch \& Strowitzki Th 1996 Morphological-cytochemical and molecular genetic analyses of mitochondria in isolated human oocytes in the reproductive age. Molecular Human Reproduction 2 951-958.

Nieder G \& Weitlauf H 1984 Regulation of glycolysis in the mouse blastocyst during delayed implantation. Journal of Experimental Zoology 231 121-129.

Potts D 1968 The ultrastructure of implantation in the mouse. Journal of Anatomy 103 77-90.

Pozzan T, Magalhaes P \& Rizzuto R 2000 The comeback of mitochondria to calcium signalling. Cell Calcium 28 279-283. 
Radisky D, Levy D, Littlepage L, Liu H, Nelson CM, Fata JE, Leake D, Godden EL, Albertson DG, Nieto MA et al. 2005 Rac1b and reactive oxygen species mediate MM-3-induced EMT and genomic instability. Nature 436 123-127.

Reers M, Smiley S, Mottola-Hartshorn C, Chen A, Lin M \& Chen L 1995 Mitochondrial membrane potential monitored by JC-1 dye. In Methods in Enzymology, vol. 260, pp 406-417. Eds G Attardi \& A Chomyn. New York: Academic Press.

Reynier P, May-Panloup P, Chretien M, Morgan CJ, Jean $M$, Savagner $\mathbf{F}$, Barrière $\mathbf{P}$ \& Malthièry $\mathbf{Y} 2001$ Mitochondrial DNA content affects the fertilizability of human oocytes. Molecular Human Reproduction 7 425-429.

Rutter G \& Rizzuto R 2000 Regulation of mitochondrial metabolism by $\mathrm{ER} \mathrm{Ca}^{2+}$ release: an intimate connection. Trends in Biochemical Sciences 25 215-220.

Salvioli A, Ardizzoni A, Franceschi C \& Cossarizza A 1977 JC-1, but not $\mathrm{DiOC}_{6}(3)$ or rhodamine 123, is a reliable fluorescent probe to assess $\Delta \Psi$ changes in intact cells: implications for studies on mitochondrial functionality during apoptosis. FEBS Letters 411 77-82.

Steuerwald N, Barrit JA, Adler R, Malter H, Schimmel T, Cohen J \& Brenner CA 2000 Quantification of mtDNA in single oocytes, polar bodies and subcellular components by real-time rapid cycle fluorescence monitored PCR. Zygote 9 209-215.

Struthers L, Patel R, Clark J \& Thomas S 1998 Direct detection of 8-oxodeoxyguanosine and 8-oxoguanine by avidin and its analogues. Analytical Biochemistry 255 20-31.

Thouas G, Trounson A, Wolvetang E \& Jones G 2004 Mitochondrial dysfunction in mouse oocytes results in preimplantation embryo arrest in vitro. Biology of Reproduction 71 1936-1942.

Trimarchi J, Liu L, Porterfield D, Smith P \& Keefe D 2000 Oxidative phosphorylation- dependent and -independent oxygen consumption by individual preimplantation mouse embryos. Biology of Reproduction 62 1866-1874.

Van Blerkom J 2004 Mitochondria in human oogenesis and preimplantation embryogenesis: engines of metabolism, ionic regulation and developmental competence. Reproduction 128 269-280.

Van Blerkom J \& Motta P 1979 In The Cellular Basis of Mammalian Reproduction, Ch 5. Baltimore, MD: Urban, Schwarzenberg.

Van Blerkom J \& Chavez DJ 1981 Morphodynamics of outgrowths of mouse trophoblast in the presence and absence of a monolayer of uterine epithelium. American Journal of Anatomy 162 143-155.

Van Blerkom J, Chavez D \& Bell H 1979 The molecular and cellular biology of facultative delayed implantation in vivo and in vitro. In Maternal Recognition of Pregnancy, pp 141-172. CIBA Symposium no. 64. Amsterdam: Excerpta Medica.
Van Blerkom J, Davis P \& Lee J 1995 ATP content of human oocytes and developmental potential and outcome after in vitro fertilization and embryo transfer. Human Reproduction 10 415-424.

Van Blerkom J, Davis P \& Alexander S 2000 Differential mitochondrial distribution in human pronuclear embryos leads to disproportionate inheritance between blastomeres: relationship to microtubular organization. ATP content and competence. Human Reproduction 15 2621-2633.

Van Blerkom J, Davis P \& Alexander S 2001 A microscopic and biochemical study of fragmentation phenotypes in stage-appropriate human embryos. Human Reproduction 16 719-729.

Van Blerkom J, Davis P, Mathwig V \& Alexander S 2002 Domains of high-polarized and low-polarized mitochondria may occur in mouse and human oocytes and early embryos. Human Reproduction 17 393-406.

Van Blerkom J, Davis P \& Alexander S 2003 Inner mitochondrial membrane potential $(\Delta \Psi \mathrm{m})$, cytoplasmic ATP content and free $\mathrm{Ca}(2+)$ levels in metaphase II mouse oocytes. Human Reproduction 18 2429-2440.

Webb S \& Miller A 2003 Calcium signalling during embryonic development. Nature Reviews. Molecular Cell Biology 4 539-551.

Weitlauf H \& Kiessling A 1980 Comparison of overall rates of RNA synthesis in implanting and delayed implanting mouse blastocysts in vitro. Biology of Reproduction 14 566-573.

Wilding M, Dale B, Marino M, di Matteo L, Alviggi C, Pisaturo ML, Lombardi L \& De Placido G 2001 Mitochondrial aggregation patterns and activity in human oocytes and preimplantation embryos. Human Reproduction 16 909-917.

Wilding $M$, Fiorentino A, de Simone $M$, Infante V, de Matteo L \& Dale B 2002 Energy substrates, mitochondrial membrane potential and human preimplantation embryo division. Reproductive Biomedicine Online 5 39-42.

Wilding M, De Placido G, di Matteo L, Marino M, Alviggi C \& Dale B 2003 Chaotic mosaicism in human preimplantation embryos is correlated with a low mitochondrial membrane potential. Fertility and Sterility 79 340-346.

Received 11 August 2005

First decision 19 October 2005

Revised manuscript received 5 December 2005

Accepted 25 January 2006 